\title{
MOMEE: Manifold Optimized Modeling of Energy Efficiency in Wireless Sensor Network
}

\author{
Rajalakshmi M.C. \\ Research Scholar \\ University of Mysore \\ Karnataka, India
}

\author{
A.P. Gnana Prakash \\ Associate Prof.: Dept. of Studies in Physics \\ University of Mysore \\ Karnataka, India
}

\begin{abstract}
Although adoption pace of wireless sensor network has increased in recent times in many advance technologies of ubiquitous-ness, but still there are various open-end challenges associated with energy efficiencies among the sensor nodes till now. We reviewed the existing research approaches towards energy optimization techniques to explore significant problems. This paper introduces MOMEE i.e. Manifold Optimized Modeling of Energy Efficiency that offers novel clustering as well as novel energy optimized routing strategy. The proposed system uses analytical modeling methodology and is found to offer better resiliency against traffic bottleneck condition. The study outcome of MOMEE exhibits higher number of alive nodes, lower number of dead nodes, good residual energy, and better throughput as compared to existing energy efficient routing approaches in wireless sensor network.
\end{abstract}

Keywords-Wireless Sensor Network; Energy Efficiency; network Lifetime; Optimization; Battery

\section{INTRODUCTION}

The concept of wireless sensor network has undergone a tremendous change in last decade. Conventional theory says that it was all about a network of uniformly or randomly distributed sensor nodes being monitored by single / multiple base station [1][2]. Such sensor nodes are quite smaller in size and have lower computational capability as well as restricted resource availability [3]. Once the sensors are deployed over the area that are required to be monitored remotely, they starts capturing the raw environmental data using Time Division Multiple Access (TDMA) and forward it to the sink or base station. This process is called as data aggregation [4]. Theoretical study and frequently used research-based study considers three types of nodes i.e. i) member node, ii) cluster head, and iii) base station as the prime actors playing crucial role in communication process. At the same time, clustering mechanism is an extremely critical concept before talking about communication. All the nodes that are dispersed in the area should be subjected to clustering mechanism so that there is proper organization of communication in order to assists in data aggregation process. Majority of the clustering mechanism carried out till date converges towards a concept of cluster head selection process, where $99 \%$ of the work done is by considering maximum residual energy as the prominent criteria towards selection of cluster head. However, this is not true and there is various research works where researchers have chosen non-energy parameters too in the selection process. At present, there are various studies being carried out towards enhancing clustering process [5], routing process [6], load balancing process [7], and traffic management process. Unfortunately, very less number of works has been found to be attained a height of standards. It is said that every sensor nodes works on the principle of 1st order radio-energy model [8][9] in order to balance energy and communication trade off. Still, optimal solution has not being received. It is known that a sensor (i.e. cluster head) is allocated a specific amount of transmittance energy during data aggregation, which is higher compared to other nodes (i.e. member nodes). It will mean that every cycle of data aggregation witnesses a declination of number of active nodes. This problem is very hard to be solved. It is because if the application of sensors calls has apriori information of incoming signals than it is somewhat feasible to control the energy consumption by an alternative measures. But majority of the sensor applications are used in a scenario where incoming traffic generation is quite uncertain (e.g. habitat monitoring, natural calamities monitoring, health monitoring, etc). This causes uneven rate of dissipation of energy per clusters during each rounds of data aggregation. Hence, majority of the research attempts fails to prove efficiency of energy as such real-time uncertainty problems are less considered. Although, there are various research work towards optimization in wireless sensor network, there is a less benchmarked work with respect to energy efficiency. This problem has multi-facet negative effect on communication performance with all forms of declinations towards Quality-ofService (QoS) parameters. The problem becomes much worst when we realize the upcoming usage of wireless sensor network in Internet-of-Things (IoT) that integrates sensors with cloud environment. This will definitely pose a massive challenges towards sensor network in order to be capable of assisting in pervasive computing e.g. IoT.

Therefore, this paper introduced a novel multi-level optimization process that retains good balance between energy consumption and optimal QoS performance. Section II discusses about the recent research work being carried out towards addressing energy consumption problem followed by problem identification in Section III. The contribution of proposed system is discussed in Section IV followed by elaboration of research methodology in Section V. Algorithm implementation is discussed in Section VI followed by result analysis. Finally, summarization of the work and findings are briefed in conclusion in Section VII and section VIII concludes future work of the research. 


\section{RELATED WORK}

Our prior study has discussed about various research techniques associated with solving the problems of energy dissipation in wireless sensor network [10]. We update some of the recent work done towards energy efficiency that is claimed to enhance the network lifetime.

Most recently, the work carried out by $\mathrm{Wu}$ et al. [11] have addressed the problem with delay as well as energy by introducing a unique optimization technique especially in flooding schemes. A routing scheme characterized by duty cycle was introduced along with enhanced minimum spanning tree. The study outcome was found to provide $20-60 \%$ of energy conservation. Kumar and Chaturvedi [12] have presented a technique of energy conservation using clusteringbased approach and fuzzy logic considering static multiple sink nodes. A very new concept of wireless power transmission was discussed by Hong et al. [13] that uses selective patterns of beam. It consists of charging and transmission state. The presented technique mainly emphasized over allocation of charging power using optimization principle considering channel state information. Latif et al. [14] have presented a study that addresses energy outage in the dense area of node deployment considering the case study of underwater sensor network. The study outcome was assessed using delay, energy, coverage, rate of packet reception. The resultants show better improvement over coverage and its direct effect on energy efficiency. Similar direction of the work was carried out by Manju et al. [15] where the problem of energy efficiency and network lifetime was discussed. A heuristic-based approach was presented for identifying the significant targets in wireless sensor network. The study outcome show better improvement of network lifetime. Nayak and Anurag have presented a technique where fuzzy logic was used for enhancing the performance of clustering in wireless sensor network. Such forms of approaches are abundant to be seen in the literatures and are highly repetitive nature. The work carried out by the Pilloni et al. [17] has also addressed energy problems in sensor network. The authors have used gossip-based distributed approach in order to perform asynchronous optimization that directly assists in energy conservation among the sensors.

The problems of energy hole has been addressed in the work carried out by Ren et al. [18] where a temporal and spatial relationship has been discussed in order to do this. Wang et al. [19] have presented a unique optimization modeling that incorporates mobility in order to overcome the communication problems in sensor network. The base station is made mobile that perform data aggregation in order to increase the rate of data aggregation and minimize the energy consumption involved in the process. A distributed algorithm is design that fine tunes provisioning of link, data rate, and perform flow routing during any forms of variance on energy. The outcome was found to offer better energy conservation assessed in both static and mobile aggregation of data. Liu et al. [20] have introduced combined channel as well as network coding technique for energy optimization. The assessment has used bit error rate as well as standard fading and noisy channel where the study outcome shows that energy required for precise decoding is highly minimized over increasing noise. Pourazarm et al. [21] have jointly discussed the problem of energy efficiency as well as security in sensor network. An interesting solution is provided by this technique which renders uniform energy dissipation using non-linear optimization technique. An allocation policy for energy was introduced by the author in this regards. Tabus et al. [22] have used linear programming as the optimization technique for enhancing the network lifetime. The technique introduces a reconfigurable chain topology in order to increase the scheduling between the base station and network. This process significantly increases energy conservation in wireless sensor network. Wan et al. [23] have presented a technique based on polynomial equation in order to enhance the network lifetime. The technique introduces a specific scheduling process for the node to switch between wake-up and sleep mode. The study outcome was found to improve the network lifetime. Adoption of cross layer based approach for the purpose of energy optimization was seen in the study of Xu et al. [24]. The authors have developed a stochastic-based modeling over discrete time along with Lyapunov factor for better optimization performance. A new energy modeling concept was introduced by the author along with a pricing model followed by formation of various objective function. Testing using multi-channel sensor network, the result shows higher backlogs and satisfactory rate achievement of the presented technique.

The process of routing is always linked with energy consumption in sensor network where various non-linear programming aspects have already been discussed in recent times. The work carried out by Cassandras et al. [25] have presented a model that emphasize on the problem of allocation of energy and routing behaviour. This work is exactly similar to the work carried out by Pourazarm et al. [21]. Demigha et al. [22] have also addressed the problem of energy optimization by introducing linear programming with binary integers. The study chooses to work under the constraint of data accuracy as it emphasize on the correlational analysis of the aggregated data by the sensor nodes. The technique also incorporates a heuristic-based solution that enhances the optimization performance. Habibi et al. [27] have discussed about significance of cooperative routing and its possible impact over network lifetime. The technique was focused on reduced complexity design for its optimization. Li et al. [28] have discussed a technique that uses multiple-input multiple-output approach using cooperative transmission. The assessments of the presented concept were studied with respect to intra and inter clustering using packet error rate. The overall performance of the system was carried out considering energy consumption. The study outcome shows that fine-tuning the clustering has positive effect over the energy consumption. Lee et al. [29] have used bio-inspired algorithms in order to enhance the network lifetime of wireless sensor network. The authors have addressed the problem of coverage and associated with energy consumption. The authors have modified the conventional Ant Colony Optimization to obtain better outcomes. Ramachandran and Sikdar [30] have utilized population matrix in order to model the enhancement framework towards network lifetime. Hence, recent research contributions do have significant contribution towards energy problems and has evolved up with various optimization plans. The next section discuss about the problems identified in the existing research work. 


\section{PROBLEM IDENTIFICATION}

This section discusses about the problems that has been explored after reviewing the existing research towards addressing the energy efficiency problems in wireless sensor network.

\section{A. More usage of Conventional Clustering}

The existing conventional technique of clustering mainly focuses on selection of cluster head on the basis of higher residual energy. However, it doesn't emphasize much on the optimal positions of the candidate nodes with respect to multiple criteria that could possibly offer optimal routing performance. Maximum focus was given on routing approaches and less on clustering causing less improvement in network lifetime.

\section{B. Less number of simpler Optimization Approaches}

Optimization is the only best alternative for performance upgrading among resource-limited sensors. However, usages of existing optimization techniques are higher recursive in nature and are definitely not proactive in nature. This results in biased performance outcome and contributes to computation and communication trade-off. A simple optimization technique not only ensures lower resource allocation but also ensures the sustenance capacity for the longer duration of time.

\section{Less Control over Energy Factor}

Existing energy efficient or energy-aware routing techniques is mainly focused on forming a new routing strategy without much work in energy modeling. The overall outcome of such routing operation however is shown to have positive effect on energy conservation among the sensor networks. In reality, it doesn't lower any forms of energy deterministically. Hence, it is quite uncertain if such technique will always maintain similar energy efficient characteristics on different conditions of traffic especially at bottleneck condition.

\section{Computation Communication Tradeoff}

At present none of the research techniques bridges the gap between communication performance and computation performance. Inclusion of higher order algorithms and iterative approaches for solving problems only enhances communication performance leading computation problems aside. A sensor node cannot process more due to its limitation of 2-4 KB of RAM size. Hence, sophisticated algorithm inclusion (normally in clustering) will result in more number of processing that cost the energy factor leading to lower number of the nodes.

Therefore, it can be seen that although there are various research work being carried out towards energy enhancement, routing, optimization etc. but majority of them suffers from the above mentioned problems that are still unsolved. Hence, the problem statement can be cited as "to develop a simple nonrecursive and manifold optimization technique that provides more control over the energy optimization on adverse traffic condition in wireless sensor network." The next section discusses about the contribution of the proposed system in the form of adopted research methodology.

\section{PRoposed Methododology}

In a continuation of our prior studies [31], [32], [33], the proposed study introduces a technique where a multiple-level of optimization is carried out for the purpose of enhancing the network lifetime of wireless sensor network. The complete work has been carried out considering analytical methodology. Our schematic architecture is shown in Fig.1. The discussion of the component blocks of the architecture with respect to the adopted methodology is illustrated in this section as follows:

\section{A. Sensor Parameter Initialization}

The implementation of the proposed study was carried out considering number of sensor adhering to the specification of MEMSIC nodes [34]. We also vary the position of the base station in order to ensure that proposed MOMEE ensures similar performance irrespective of any position of the base station. Inclusion of base station was carried out as majority of the mechanism consider base station either outside of simulation area or is fixed on a particular area of simulation. Hence, in order to break this myth of base station uniform location, we keep it variable. The study also considers packet length as well as initialized energy.

\section{B. Algorithm for Clustering}

Majority of the existing mechanism of clustering is carried out on the basis of higher residual energy. In compliance with standard clustering, we also choose probability of certain nodes to be become cluster head and perform computation of the cardinality of clusters to be formed in the entire cycle of data aggregation. We offer a novel empirical approach to compute cluster cardinality, which cannot be seen in any existing clustering mechanism. Apart from this, we construct a matrix that has apriori information of the cluster heads and keeps on updating after successful completion of data aggregation. This offers faster selection process of cluster head in next cycle in order not to disrupt the pace of data transmission. At the same time, cluster head is also selected on the basis of the superior positioning of candidate nodes. Not all the nodes become cluster head in our approach. The advantage of this methodology is that MOMEE offers significant support to multihop routing where decision of stabilized routing should take place faster.

\section{First Order Radio Energy Model}

It was necessary to ensure complete compliance of proposed energy modeling with respect to first order radioenergy model in wireless sensor network. The primary advantage of adopting this modeling is that comparative analysis with any existing hierarchical routing protocol becomes easier. Moreover the elementary concept of first order radio model significant helps in ensuring the fact that proposed simulation-based study is in adherence to near real-time sensory applications in wireless sensor network. Testifying the effectiveness of MOMEE with respect to hardware circuit components and antenna management becomes easier when the design principle is in the order to first order radio-energy model in wireless sensor network. 


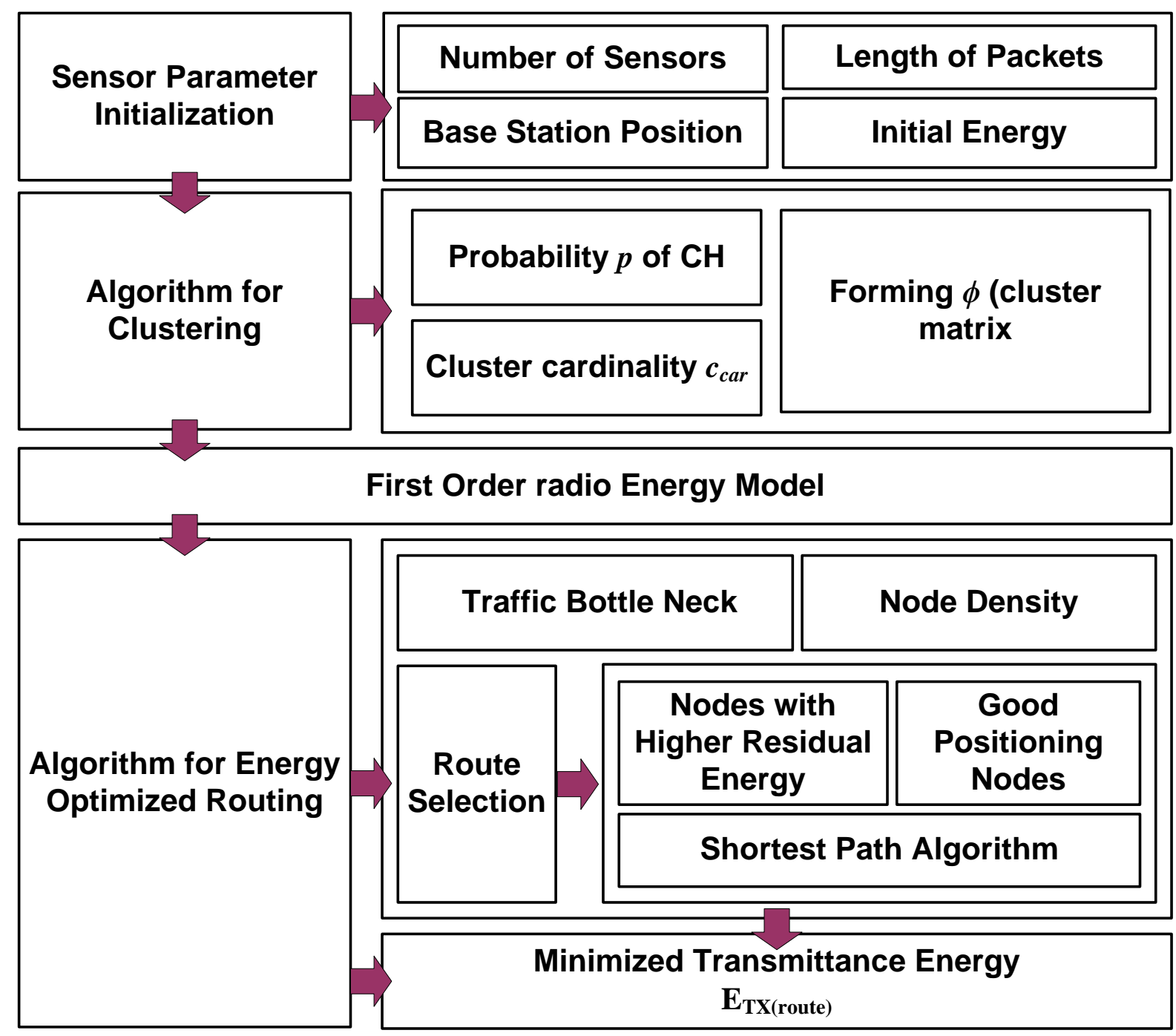

Fig. 1. Schematic Architecture of MOMEE

\section{Algorithm for Energy Optimized Routing}

Majority of the existing approaches offers communication in energy optimized path and for that an algorithm is written to explore such path. But different from existing approach, we not only explore such path but also offer two novel contribution viz. i) incorporate manifold optimization and ii) reduce the transmittance energy. This methodology too cannot be seen to be attempted on any prior research technique. From the viewpoint of resource allocation, we strongly believe that transmittance energy of cluster head is quite higher than other nodes (member and candidate). At the same time, it is not directly possible to lower the transmittance energy if the packet is bigger in size. Hence, we introduce traffic bottleneck as well as node density upon whose presence a perfect route has to be selected. Using the proposed principle of manifold optimization, we ensure that a route is selected on multiple criteria e.g. nodes with higher residual energy, good positioning of nodes, and shortest path algorithm. The good position of the node will mean the best position which will cause lower overhead in communication, is near to base station, and have sufficient residual energy compared to its adjacent nodes. Apart from this, the proposed mechanism also offers a random orientation as a fast route finder handler in order to find congestion free routes and takes binary decision of either opting or rejecting the considered selected path. Hence, our proposed mechanism has non-recursive optimization step with better and practical logic for selection of path. Not only this, once the path is selected, we develop an empirical operator that minimizes the transmittance energy to a very large extent. Hence, the objective function created for this purpose itself ensures that i) MOMEE must perform multi-criteria selection of stabilized routes and ii) only the routes will be chosen that offer reduction possibility of transmittance energy.

Therefore, it can be now seen that proposed MOMEE offers a comprehensive scheme of power and routing management that is mainly meant for enhancing the network lifetime. Adherence to first order radio energy model is also another reason of higher applicability of proposed system on sensory application that demands more power and needed to be operated in adverse environment for longer duration. The next section discusses about algorithm implementation. 


\section{ALGORITHM IMPLEMENTATION}

This section discusses about the algorithm that has been used for the purpose of implementing proposed MOMEE concept in wireless sensor network. Basically, there are two prominent algorithms responsible for incorporating the concept of manifold optimization i.e. i) Algorithm for Clustering and ii) Algorithm for Energy Optimized Routing. Both the algorithms considers traffic bottleneck as the vital traffic condition arising from uncertainty problem in traffic management by the sensor nodes.

\section{A. Algorithm for Clustering}

The prime purpose of this algorithm is to offer a novel mechanism of clustering. This algorithm takes the input of $E$ (initialized energy), $n$ (sensors), $c_{c a r}$ (cluster cardinality), $p$ (probability), and $B$ (Boundary) which after processing leads to the generation of $\phi$ (cluster matrix). The steps involved in the algorithm are as follows:

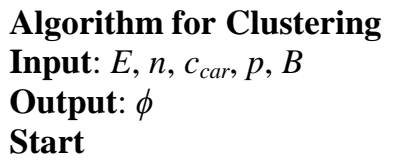

1. init $\mathrm{E}, \mathrm{n}(\operatorname{rand}(\mathrm{x}, \mathrm{y}))$

2. $\mathrm{c}_{\mathrm{car}} \rightarrow\left|\mathrm{z}^{2}-\mathrm{n} . \mathrm{p}\right|$, where $\mathrm{z}=1,2, \ldots$

3. construct $\phi=f[\phi, \sqrt{\phi}], \phi \Rightarrow\left[\arg _{\min }\left(c_{c a r}\right)\right]^{2}$

4. For $i=1: n$

5. $\left[\mathrm{x}_{\text {temp }}, \mathrm{y}_{\text {temp }}\right] \rightarrow[\mathrm{x}(\mathrm{i}), \mathrm{y}(\mathrm{i})]$

6. If $\left[\mathrm{x}_{\mathrm{temp}}, \mathrm{y}_{\mathrm{temp}}\right]>\mathrm{B}[\mathrm{i}] \& \&\left[\mathrm{x}_{\mathrm{temp}}, \mathrm{y}_{\mathrm{temp}}\right]<\mathrm{B}[\mathrm{i}+1]$

7. Flag $\phi\left(\mathrm{x}_{\mathrm{i}}, \mathrm{y}_{\mathrm{i}}\right) / /$ cluster ID

8. End

9. End

End

The algorithm performs initialization of the energy with respect to first order radio-energy model (Line-1). At the same time, all the sensors are randomly dispersed within the simulation area (Line-1). The position of the base station could be anywhere within the simulation area. We define the cardinality of the cluster heads $c_{c a r}$ using two parameters i.e. i) an integer $z$ and ii) rounded number of cluster heads computed as product of sensors $n$ and probability $p$ (Line-2). This decision of clustering is different compared to any existing system as cumulative clusters $\phi$ are decided depending on the alive nodes only as well as probability (Line-3). For costeffectiveness, we compute the clusters with minimum arguments and thereby formulate a final cluster matrix $\phi$ (Line$3)$. A manifold optimization is implemented by creating a squared matrix of size square-root $\phi$ and its elements are formed column wise from. The process of new positions of the nodes $\left(x_{\text {temp }}, y_{\text {temp }}\right)$ is checked (Line-5) for all the sensors (Line4) and the cluster is formulated on this basis. It is also ensured that only the nodes within the boundary B will be chosen for clustering mechanism (Line-6). This information is stored as a cluster Id in final cluster matrix $\phi$ (Line-7), which will be updated on the basis of cycle of data aggregation and formation of new clusters in the progressive process. Hence, all the clusters formulated can be said to be well positioned and all the cluster head have good amount of residual energy. Hence, we don't perform clustering only on the basis of residual energy but also on the basis of well-defined position where communication vector and its frequencies of data transmission is higher. The purpose is to maintain balance between increased communication performance and controlled manner of energy dissipation. However, algorithm for clustering is only focused on clustering while algorithm for optimized routing is mainly responsible for minimizing energy dissipation.

\section{B. Algorithm for Energy Optimized Routing}

This algorithm is primarily responsible for finding the energy efficient routes. The significant contribution of this algorithm is that it performs minimization of reduced transmittance energy. The primary input for this algorithm is $\tau$ (traffic bottleneck) as well as it also uses $\gamma$ (Signum function) which upon processing results in $E_{T X(\text { route) }}$ (Energy Efficient Route). The steps involved in the algorithm are as follows:

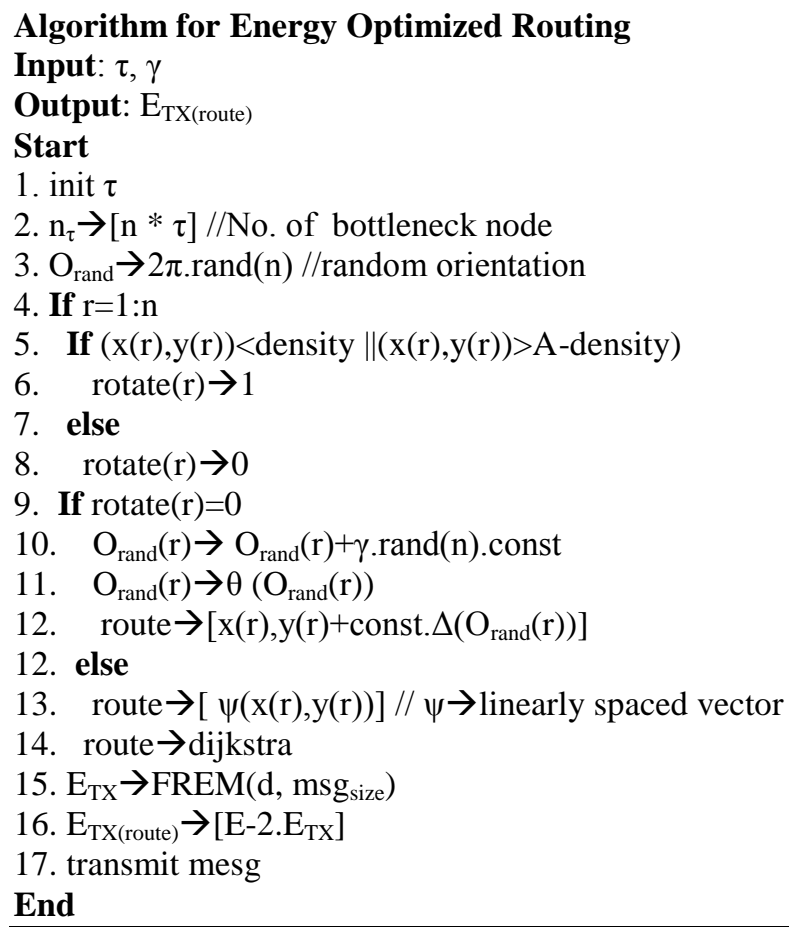

The algorithm first initializes $\tau$ (traffic bottleneck) (Line-1) and computes number of the sensors suffering from traffic bottleneck. So, we represent $n_{\tau}$ as the number of bottleneck sensors (Line-2). A random orientation is formulated in Line-3 in order to find the best node to be selected for upcoming routing mechanism (for data aggregation). An argument $r$ is formed that considers all nodes (Line-4) and checks of the new position of the node have higher node density (Line-5). For positive case of traffic (with low density), a new path is selected for the next neighbor nodes (Line-7) or else it is rejected (Line-8). As a part of manifold optimization, we further add more random parameters with Signum function in order to offer the node a positive or negative direction of routing (Line-9-Line-10). A function of angle $\theta$ is formulated which offers condition if the angle of communication vector is more than or less than $2 \pi$ (Line-11). If the angle is found to be more than $2 \pi$, we subtract the angle with $2 \pi$ or else we add it. Finally, a route is selected on the basis of this new position of the nodes. We also use network related constants const and $\Delta$ 
for further adding a new layer of optimization (Line-12). Hence, Line-10-12 will be executed for the condition of there is no rotation (i.e. for static nodes). However, for mobile nodes, we use linearly spaced vector $\psi$ among the existing and new position of the nodes to form a communication path (Line-13). We also apply shortest path algorithm to form this route (Line14) as well as we apply First Order Radio-Energy Model (FREM) considering Euclidean distance and size of message in order to compute transmitted energy (Line-15). We further minimize the transmittance energy of cluster head $\mathrm{E}_{\mathrm{TX}}$ by subtracting it with twice of $\mathrm{E}_{\mathrm{TX}}$ computed on the selected route (Line-16) and then transmit the message (Line-17).

Hence, the proposed system offers comprehensive steps in order to perform clustering based on superior node position and then performs routing on the path that are free from bottlenecks and more stabilized nodes (nodes with higher threshold residual energy). The next section discusses about the outcome accomplished from the proposed study of MOMEE.

\section{RESULT ANALYSIS}

This section discusses about the outcomes accomplished from the proposed implementation of MOMEE. We consider 100 sensors dispersed randomly in $1200 \times 1500 \mathrm{~m}^{2}$ of simulation area. The base station can have variable position within the simulation area with 0.05 probability value of cluster head, 0.5 Joule as initial energy, 2000 bits as packet length, and 0.2 as traffic bottleneck. All these variables can be amended to offer an increasing scope of assessment of proposed system. In order to perform assessment of its effectiveness, we consider comparing the outcome of the proposed MOMEE with conventional LEACH algorithm [35] that offers standard energy efficiency. We also choose to compare with the most recent work being carried out by Siavoshi et al. [36]. The reason behind considering this work is because i) similar objective of multi-level optimization technique and ii) similar goal of energy efficiency. The authors have presented a protocol that offers multi-layered clustering of distributed nature and minimizes the operations during intra-clustering in order to minimize the residual energy of the nodes. It was noticed that selection of the cluster head was completely carried out on the basis of the residual energy. It also performs selection of next hop and its study outcome was found to be better than LEACH. We do minor change the values of simulation variables in order to retain similar parameters to be used in LEACH, Siavoshi et al. [36] and proposed MOMEE. On 2500 simulation rounds, we choose to consider number of alive nodes, dead nodes, residual energy, and throughput as the prime performance parameter for this comparative analysis. All the outcomes were collected only after the entire network has witnessed complete node death for all the considered techniques.

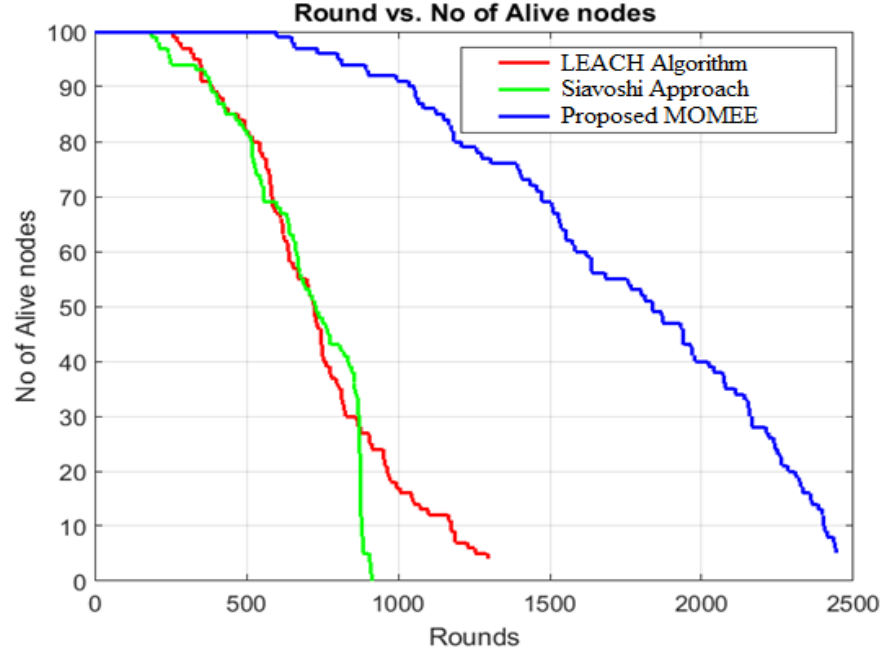

Fig. 2. Number of Alive Nodes

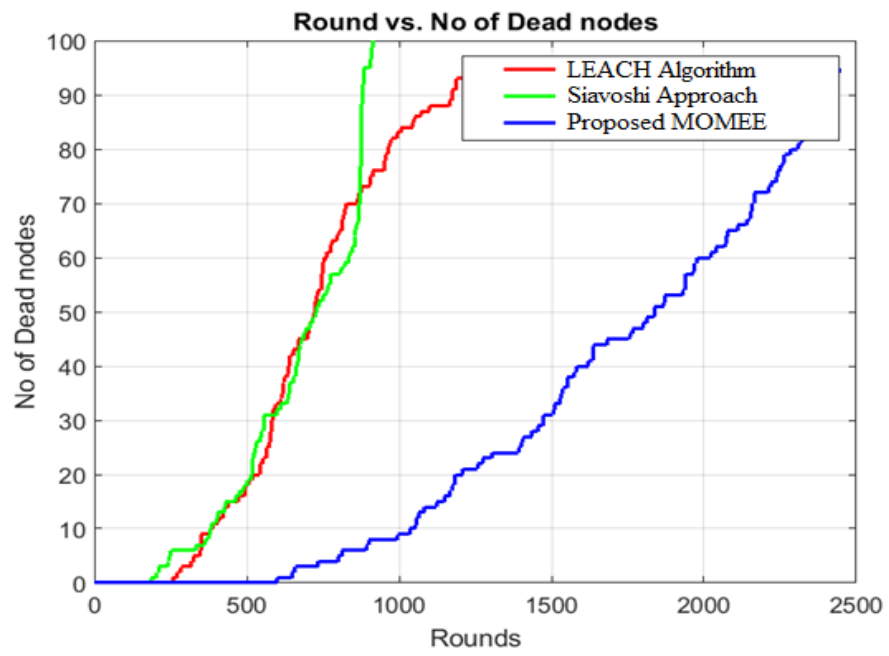

Fig. 3. Number of Dead Nodes

Fig.2 and Fig.3 shows the comparative performance analysis where it can be seen that proposed system offers better retention of nodes with good residual energy compared to existing system. Although the work carried out by Siavoshi et al. [36] has discussed that their protocol to be better than LEACH, but we don't find much significant difference if we consider the higher node density with traffic bottle necks. The prime reason behind this is the approach of Siavoshi et al. [36] offers too much rule-base checks in order to perform clustering which an added operation is carried out by every node causing higher depletion of energy. However, in presence of dense network and variable traffic bottle necks, the energy depletion is nearly same for which both LEACH and Siavoshi et al. [36] 
couldn't last more than 1500 rounds. On the other hand, we perform all such operation in the form of clustering matrix causing $90 \%$ lower load of updating, querying, by any node during routing. Hence, routing is not only faster but also maintain computation and communication balance resulting in good number of sensor nodes with enhanced network lifetime.

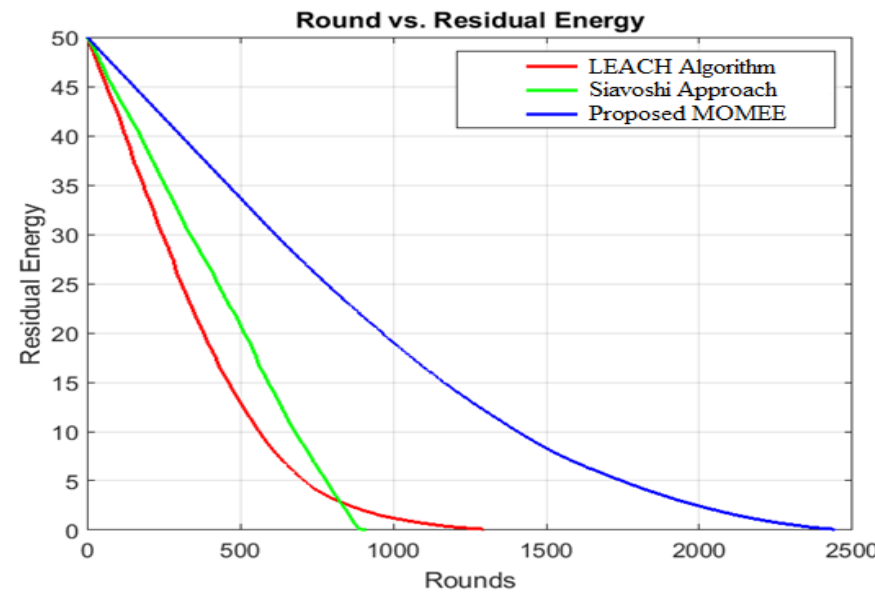

Fig. 4. Residual Energy Analysis

A closer look into Siavoshi et al. [36] approach shows better than LEACH till certain extent but owing to usage of apriori rules the declination of the residual energy is almost linear causing faster node death. As LEACH doesn't have such usage of rule sets so it manages to retain good residual energy till 1400 simulation rounds. However, it degrades faster energy as all the node (candidate head) irrespective of their position becomes cluster head resulting in node death. The process takes faster momentum in case of dense traffic condition. Proposed MOMEE offers a comprehensive policy where the reduction of transmittance energy takes place at any cost and this feature cannot be found in any existing energy-efficient routing in wireless sensor network. Hence, proposed MOMEE offers better residual energy in comparison with conventional baselines (i.e. LEACH and Siavoshi Approach).

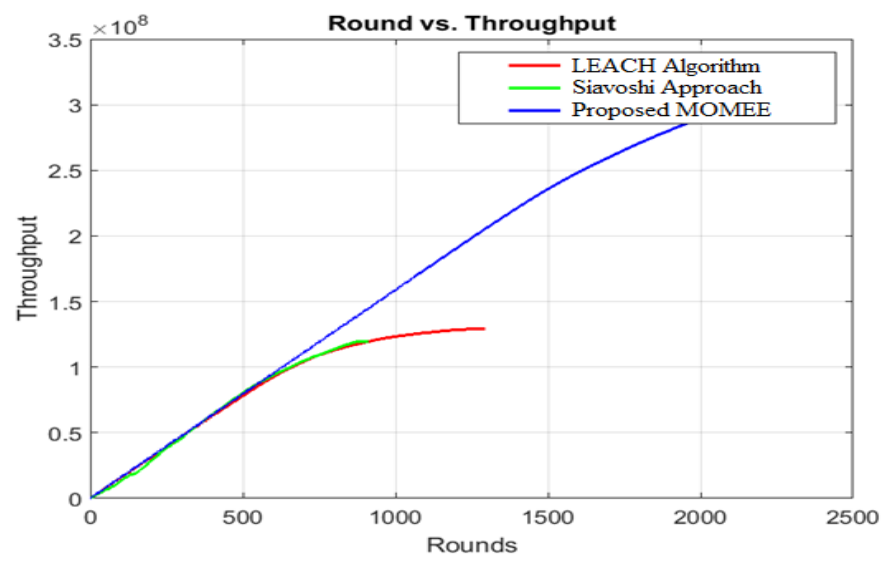

Fig. 5. Throughput Analysis

Proposed MOMEE also uses manifold optimization where the routing is selected based on i) energy, ii) superior positioning of nodes, and iii) shortest path. Therefore, it offers sustainable throughput as seen in Fig.5 compared to existing techniques. Usage of cluster matrix offers faster exploration of energy efficient routes resulting in consistently increased throughput.

\section{CONCLUSION}

Wireless sensor network has been increasingly used in modern times for remote monitoring system. Unfortunately for the lower computational and communication capabilities, the existing research work towards energy efficiencies has not gained peak success factor. Still now, when sensors are actively considered in IoT there is a problem of energy dissipation that is still unsolved apart from longer list of other problems associated with wireless sensor network. The proposed system offers following contribution / novelty viz. i) MOMEE is characterized by a novel clustering mechanism where apriori cluster formation takes place in order to lower down the response time of selecting new cluster head at the end of every data aggregation round. ii) MOMEE offers multiple nonrecursive optimization steps that not only assists in exploring stabilized links but also confirms precise delivery assurance. This characteristics is highly helpful in mission and time critical data delivery process in wireless sensor network, iii) MOMEE ensures multilevel optimization, showing compliance toward first order radio-energy model, and offers good balance between communication and energy performance.

\section{FUTURE WORK}

The future work concludes integration of the proposed MOMEE algorithm for better enhancement of energy optimal route formations by satisfying an optimal trade-off between residual energy consumptions and throughput.

\section{REFERENCES}

[1] M. Ilyas, Sami S. Alwakeel, M. M. Alwakeel, el-Hadi M. Aggoune, Sensor Networks for Sustainable Development, CRC Press, Science, 2014

[2] S. S. Iyengar, K. G. Boroojeni, N. Balakrishnan, Mathematical Theories of Distributed Sensor Networks, Springer, Technology \& Engineering, 2014

[3] B. Candaele, D. Soudris, I. Anagnostopoulos, Trusted Computing for Embedded Systems, Springer - Technology \& Engineering, 2014

[4] S. Khan, A. K. Pathan, N. A. Alrajeh, Wireless Sensor Networks: Current Status and Future Trends, CRC Press, Computers, 2016

[5] M. M. Zanjireh and H. Larijani, "A Survey on Centralised and Distributed Clustering Routing Algorithms for WSNs," IEEE 81st Vehicular Technology Conference (VTC Spring), Glasgow, pp. 1-6, 2015

[6] J. J. Lotf, M. Hosseinzadeh and R. M. Alguliev, "Hierarchical routing in wireless sensor networks: a survey," IEEE- $2^{\text {nd }}$ International Conference on Computer Engineering and Technology, Chengdu, 2010, pp. V3-650V3-654, 2010

[7] A. Krishnakumar and V. Anuratha, "Survey on energy efficient loadbalanced clustering algorithm based on variable convergence time for wireless sensor networks," IEEE 3rd International Conference on Advanced Computing and Communication Systems (ICACCS), Coimbatore, pp. 1-5, 2016

[8] R.K. yadav, V. Kumar, R.Kumar, "A Discrete Particle Swarm Optimization Based Clustering Algorithm for Wireless Sensor Networks", Springer-Emerging ICT for Bridging the Future Proceedings of the 49th Annual Convention of the Computer Society of India CSI, Vol.2, Vol.338, Series Advances in Intelligent Systems and Computing pp 137-144, 2015

[9] Munir, Kashif, Security Management in Mobile Cloud Computing, IGI Global, Computers, 2016 
[10] M.C. Rajalakshmi, “ Review of Typical Power Conservation Techniques in Wireless Sensor Network", International Journal of Computer Applications (0975 - 8887), Vol.88, No.10, February 2014

[11] S. Wu, J. Niu, W. Chou and M. Guizani, "Delay-Aware Energy Optimization for Flooding in Duty-Cycled Wireless Sensor Networks," IEEE Transactions on Wireless Communications, vol. 15, no. 12, pp. 8449-8462, Dec. 2016.

[12] P. Kumar and A. Chaturvedi, "Spatio-temporal probabilistic query generation model and sink attributes for energy-efficient wireless sensor networks," IET Networks, vol. 5, no. 6, pp. 170-177, 112016.

[13] Y. W. P. Hong, T. C. Hsu and P. Chennakesavula, "Wireless Power Transfer for Distributed Estimation in Wireless Passive Sensor Networks," IEEE Transactions on Signal Processing, vol. 64, no. 20, pp. 5382-5395, Oct.15, 152016

[14] K. Latif, N. Javaid, A. Ahmad, Z. A. Khan, N. Alrajeh and M. I. Khan, "On Energy Hole and Coverage Hole Avoidance in Underwater Wireless Sensor Networks," IEEE Sensors Journal, vol. 16, no. 11, pp. 44314442, June1, 2016.

[15] Manju, S. Chand and B. Kumar, "Maximising network lifetime for target coverage problem in wireless sensor networks," IET Wireless Sensor Systems, vol. 6, no. 6, pp. 192-197, 122016.

[16] P. Nayak and A. Devulapalli, "A Fuzzy Logic-Based Clustering Algorithm for WSN to Extend the Network Lifetime," IEEE Sensors Journal, vol. 16, no. 1, pp. 137-144, Jan.1, 2016.

[17] V. Pilloni, M. Franceschelli, L. Atzori and A. Giua, "Deployment of Applications in Wireless Sensor Networks: A Gossip-Based Lifetime Maximization Approach," IEEE Transactions on Control Systems Technology, vol. 24, no. 5, pp. 1828-1836, Sept. 2016.

[18] J. Ren, Y. Zhang, K. Zhang, A. Liu, J. Chen and X. S. Shen, "Lifetime and Energy Hole Evolution Analysis in Data-Gathering Wireless Sensor Networks," IEEE Transactions on Industrial Informatics, vol. 12, no. 2, pp. 788-800, April 2016.

[19] C. Wang, S. Guo and Y. Yang, "An Optimization Framework for Mobile Data Collection in Energy-Harvesting Wireless Sensor Networks," IEEE Transactions on Mobile Computing, vol. 15, no. 12, pp. 2969-2986, Dec. 12016.

[20] X. Liu, N. Xiong, W. Li and Y. Xie, "An Optimization Scheme of Adaptive Dynamic Energy Consumption Based on Joint NetworkChannel Coding in Wireless Sensor Networks," IEEE Sensors Journal, vol. 15 , no. 9, pp. 5158-5168, Sept. 2015

[21] S. Pourazarm; C. Cassandras, "Energy-based Lifetime Maximization and Security of Wireless Sensor Networks with General Non-ideal Battery Models," IEEE Transactions on Control of Network Systems, vol.PP, no. 99, pp.1-1

[22] V. Tabus, D. Moltchanov, Y. Koucheryavy, I. Tabus and J. Astola, "Energy efficient wireless sensor networks using linear-programming optimization of the communication schedule," Journal of Communications and Networks, vol. 17, no. 2, pp. 184-197, April 2015.

[23] X. Wan, J. Wu and X. Shen, "Maximal Lifetime Scheduling for Roadside Sensor Networks With Survivability k," IEEE Transactions on Vehicular Technology, vol. 64, no. 11, pp. 5300-5313, Nov. 2015.
[24] W. Xu, Y. Zhang, Q. Shi and X. Wang, "Energy Management and Cross Layer Optimization for Wireless Sensor Network Powered by Heterogeneous Energy Sources," IEEE Transactions on Wireless Communications, vol. 14, no. 5, pp. 2814-2826, May 2015.

[25] C. G. Cassandras, T. Wang and S. Pourazarm, "Optimal Routing and Energy Allocation for Lifetime Maximization of Wireless Sensor Networks With Nonideal Batteries," IEEE Transactions on Control of Network Systems, vol. 1, no. 1, pp. 86-98, March 2014

[26] O. Demigha, W. K. Hidouci and T. Ahmed, "A Novel BILP Model for Energy Optimization Under Data Precision Constraints in Wireless Sensor Networks," IEEE Communications Letters, vol. 18, no. 12, pp. 2185-2188, Dec. 2014

[27] J. Habibi, A. Ghrayeb and A. G. Aghdam, "Energy-Efficient Cooperative Routing in Wireless Sensor Networks: A Mixed-Integer Optimization Framework and Explicit Solution," IEEE Transactions on Communications, vol. 61, no. 8, pp. 3424-3437, August 2013.

[28] B. Li, W. Wang, H. Li, Q. Yin, Y. Zhang and H. Liu, "Performance analysis and optimization for energy-efficient cooperative transmission in random wireless sensor network," IEEE International Conference on Communications, Budapest, 2013, pp. 1635-1639.

[29] J. W. Lee, B. S. Choi and J. J. Lee, "Energy-Efficient Coverage of Wireless Sensor Networks Using Ant Colony Optimization With Three Types of Pheromones," IEEE Transactions on Industrial Informatics, vol. 7, no. 3, pp. 419-427, Aug. 2011

[30] K. Ramachandran and B. Sikdar, "A population based approach to model the lifetime and energy distribution in battery constrained wireless sensor networks," IEEE Journal on Selected Areas in Communications, vol. 28, no. 4, pp. 576-586, May 2010

[31] M.C. Rajalakshmi, A.P Gnana Prakash, "Energy Optimization for Large Scale Wireless Sensor Network using Real-Time Dynamics", International Journal of Computer Applications, Vol.108, No.7, December 2014

[32] M.C. Rajalakshmi, A.P. Gnana Prakash, "MLO: Multi-Level Optimization to Enhance the Network Lifetime in Large Scale WSN", Springer-Emerging Research in Computing, Information, Communication and Applications, pp 265-271, 2015

[33] M.C. Rajalakshmi and Gnana Prakash A P, "REEDA: Routing with energy efficiency data aggregation in wireless sensor network," IEEE International Conference on Emerging Research in Electronics, Computer Science and Technology (ICERECT), Mandya, pp. 174-179, 2015

[34] "Wireless Sensor Networks", http://www.memsic.com/wireless-sensornetworks/, Retrieved 30th Dec-2016

[35] Heinzelman, W., Chandrakasan, A., and Balakrishnan, H., "EnergyEfficient Communication Protocols for Wireless Microsensor Networks", Proceedings of the 33rd Hawaaian International Conference on Systems Science (HICSS), January 2000.

[36] S. Siavoshi, Y. S. Kavian, M. Tarhani and H. F. Rashvand, "Geographical multi-layered energy-efficient clustering scheme for ad hoc distributed wireless sensor networks," IET Wireless Sensor Systems, vol. 6, no. 1, pp. 1-9, 22016 